\title{
GENERALIZED RESONANCE MODES IN FERROMAGNETIC SPHERES
}

\author{
P. A. VOLTAIRAS, C. V. MASSALAS \\ Department of Mathematics, University of Ioannina, Ioannina GR 451 10, Greece \\ I. E. LAGARIS \\ Department of Physics, University of Ioannina, Ioannina GR 451 10, Greece
}

(Communicated by E. S. ŞUHUBI)

\begin{abstract}
In the present work we deal with the resonance modes in small ferromagnetic spheres. The analysis is based on the theory of micromagnetism, proposed by W. F. Brown and an optimization technique. Numerical results are presented for the resonance field, as well as for the resonance modes and the magnetization configuration in the material. The resonance modes described in this work could be named generalized modes.
\end{abstract}

\section{INTRODUCTION}

Magnetic resonance phenomena in magnetitite $\left(\mathrm{Fe}_{3} \mathrm{O}_{4}\right)$ have been investigated by Bickford [1] and others in the late 1950s and early 1960s. Rajagopalan and Furdyna [2] studied magnetic resonances in $\mathrm{Fe}_{3} \mathrm{O}_{4}$ spheres by microwave magnetotransmission experiments and they observed, in addition to ferromagnetic resonances, new size-dependent ones. In Ref. [2] these new resonances have been studied as a function of sphere diameter and microwave frequency. It is noted that the diameter of spheres considered in Ref. [2], was of the order of $\mathrm{mm}$ and the behaviour of the new resonances could not be described in terms of ordinary Walker modes [3]. Rajagopalan and Furdyna named these new resonances dimensional ones because of their dependence on the sphere diameter. Such spheres are obviously too large for the exchange forces to play any significant role in these resonances. Nowadays there are techniques for making very small ferromagnetic spheres $[4,5]$ which should eventually lead to resonance measurements in a size range where the exchange contributions are important. Aharoni [6] studied the possible effect of the exchange forces on the resonance modes in sufficiently small ferromagnetic particles. He offered a mechanism for some sort of size -dependent resonances in small spheres, by neglecting the magnetostatic energy and named these resonance modes exchange resonance modes as an analogue to the magnetostatic ones.

In the present work we deal with the solution of the mathematical problem describing the general theory of resonance in ferromagnetic spherical particle under the assumption of cylindrical symmetry, introduced by Aharoni [6]. The proposed analysis, in comparison to that used by Aharoni [7], has the advantage of computing the eigenvectors of the problem and it is therefore possible to plot the shape of the magnetization configuration in the material. We reduced our problem to minimizing a suitable objective function that guarantees the satisfaction of the boundary conditions. We used the optimization package MERLIN-2.1 [8,9]. Finally numerical results are presented for the resonance field and the associated shape of the magnetization configuration and the role of the particle size and frequency on the resonance field is discussed. The shape of the magnetization configuration, as it appears, could be named generalized curling mode. 


\section{PROBLEM FORMULATION}

The general theory of resonance in a ferromagnetic particle, in the absence of losses, is described by the following equation $[10,11]$

$$
\frac{\mathrm{d} \mathbf{v}}{\mathrm{d} t}=\gamma_{0} \mathbf{v} \times \mathbf{H}_{e f f}
$$

where

$$
\mathbf{H}_{e f f}=\frac{C}{M_{s}} \nabla^{2} \mathbf{v}-\frac{1}{M_{s}} \frac{\partial \omega_{\alpha}}{\partial \mathbf{v}}+\mathbf{H}
$$

is the effective field $(\partial / \partial \mathrm{v})_{i}=\partial / \partial v_{i}(i=x, y, z), \mathrm{v}$ is a unit vector parallel to the magnetization, $C=2 A$ is the exchange constant, $\omega_{\alpha}$ is the anisotropy energy density, $M_{s}$ is the saturation magnetization, $t$ is the time, $\gamma_{0}$ is the gyromagnetic ratio and $\mathbf{H}=\mathbf{H}_{0}+\mathbf{H}_{m}$ is the magnetic field which is composed of the applied field $\mathbf{H}_{0}$, and that, $\mathbf{H}_{m}$, created by the volume and surface charge of the magnetiziation distribution. The boundary conditions for the set of equations (2.1) are

$$
\frac{\partial \mathbf{v}}{\partial n}=\mathbf{0}
$$

where $\partial / n=v \cdot \nabla$ and $n$ denotes the unit outward normal to the particle surface. To the equations (2.1-3) we have to add the equations for the potential problem which in the present case are [12]

$$
\nabla^{2} V_{i n}=4 \pi M_{s} \nabla \cdot \mathrm{v}
$$

inside the particle and

$$
\nabla^{2} V_{\text {out }}=0
$$

outside it. The boundary conditions on particle surface are

$$
\begin{gathered}
V_{\text {in }}=V_{\text {out }} \\
-\frac{\partial V_{\text {in }}}{\partial n}+4 \pi M_{s} v_{n}=-\frac{\partial V_{\text {out }}}{\partial n}
\end{gathered}
$$

where $v_{n}=\mathbf{n} \cdot \mathbf{v}$. In the experimental studies of resonances, a large dc field $\mathbf{H}_{0}$ is applied; its direction is identified here with the $z$-axis. The field $\mathbf{H}_{0}$ keeps the magnetization almost parallel to the $z$-axis so that $v_{x}$ and $v_{y}$ are small. To a first order in these small quantities, the differential equations (2.1) for a steady-state solution, ( $) e^{i \omega t}$, become

$$
\begin{aligned}
& \left(\frac{C}{M_{s}} \nabla^{2}-H_{z}\right) v_{x}-\left(\frac{i \omega}{\gamma_{0}}\right) v_{y}=\frac{\partial V_{i n}}{\partial x} \\
& \left(\frac{C}{M_{s}} \nabla^{2}-H_{z}\right) v_{y}+\left(\frac{i \omega}{\gamma_{0}}\right) v_{x}=\frac{\partial V_{i n}}{\partial y}
\end{aligned}
$$

where $V_{i n}$ is the potential due to the transverse magnetization $m=M_{s}\left(v_{x} \mathbf{i}+v_{y} \mathbf{j}\right)$ and $\omega$ is the resonance frequency. In order a confusion to be avoided, we note here that we keep the same symbols for the time independent components of $\mathbf{v}$ and for the potentials. The potential due to $z$-component is included in $H_{z}$. In the case under discussion (spherical particle) $H_{z}$ includes the dc field $H_{0}$, the demagnetizing field

$$
H_{D}=-\frac{4 \pi}{3} M_{s}
$$


and the anisotropy field $H_{k}$, that is

$$
H_{z}=H_{0}-\frac{4 \pi}{3} M_{s}+\frac{2 K_{1}}{M_{s}}
$$

where $K_{1}$ is the anisotropy constant. It is noted that for the above linearized equations cubic or uniaxial anisotropies lead to the same expression provided that $z$ is an easy axis.

\section{PROBLEMS SOLUTION}

Following Aharoni [6] we suppose that $\mathbf{v}$ does not depend on the coordinate $\phi$ (cylindrical symmetry) and use the components of $\mathbf{v}$ in a cylindrical coordinate system $(\varrho, \phi, z)$ but express the spatial dependence in spherical coordinates $(r, \theta, \phi)$. Under these considerations the equations (2.8) and (2.9) are transformed as

$$
\begin{gathered}
\left(\frac{\partial^{2}}{\partial r^{2}}+\frac{2}{r} \frac{\partial}{\partial r}+\frac{2}{r^{2}} \frac{\partial^{2}}{\partial \theta^{2}}+\frac{\cos \theta}{r^{2} \sin \theta} \frac{\partial}{\partial \theta}-\frac{1}{r^{2} \sin ^{2} \theta}-\frac{M_{s} H_{z}}{C}\right) v_{\phi}+\frac{i M_{s} \omega}{\gamma_{0} C} v_{\rho}=0 \\
\left(\frac{\partial^{2}}{\partial r^{2}}+\frac{2}{r} \frac{\partial}{\partial r}+\frac{2}{r^{2}} \frac{\partial^{2}}{\partial \theta^{2}}+\frac{\cos \theta}{r^{2} \sin \theta} \frac{\partial}{\partial \theta}-\frac{1}{r^{2} \sin ^{2} \theta}-\frac{M_{s} H_{z}}{C}\right) v_{\rho}-\frac{i M_{s} \omega}{\gamma_{0} C} v_{\phi} \\
=\frac{M_{s}}{C}\left(\sin \theta \frac{\partial}{\partial r}+\frac{\cos \theta}{r} \frac{\partial}{\partial \theta}\right) V_{i n},
\end{gathered}
$$

respectively. The equations (2.4) and (2.5) of the potential problem are written as

$$
\left(\frac{\partial^{2}}{\partial r^{2}}+\frac{2}{r} \frac{\partial}{\partial r}+\frac{2}{r^{2}} \frac{\partial^{2}}{\partial \theta^{2}}+\frac{\cos \theta}{r^{2} \sin \theta} \frac{\partial}{\partial \theta}\right) V_{i n}=4 \pi M_{s}\left(\frac{1}{r \sin \theta}+\frac{\cos \theta}{r} \frac{\partial}{\partial \theta}+\sin \theta \frac{\partial}{\partial r}\right) v_{o}
$$

for $r \leq R$, where $R$ is the radius of the spherical particle, and

$$
\left(\frac{\partial^{2}}{\partial r^{2}}+\frac{2}{r} \frac{\partial}{\partial r}+\frac{2}{r^{2}} \frac{\partial^{2}}{\partial \theta^{2}}+\frac{\cos \theta}{r^{2} \cos \theta} \frac{\partial}{\partial \theta}\right) V_{o u t}=0
$$

for $r \geq R$. The boundary conditions (2.3), (2.6) and (2.7) on $r=R$ become:

$$
\begin{gathered}
\frac{\partial v_{\varrho}}{\partial r}=\frac{\partial v_{\phi}}{\partial r}=0 \\
V_{\text {in }}=V_{\text {out }}
\end{gathered}
$$

and

$$
\frac{\partial V_{\text {in }}}{\partial r}-\frac{\partial V_{\text {out }}}{\partial r}=4 \pi M_{s} v_{Q} \sin \theta
$$

respectively. Expanding the solution of the problem in a series of Legendre functions

$$
\begin{gathered}
v_{e}=\sum_{n=1}^{\infty} A_{n}(r) P_{n}^{1}(\cos \theta) \\
v_{\phi}=i \sum_{n=1}^{\infty} B_{n}(r) P_{n}^{1}(\cos \theta) \\
V_{\text {in }}=\sum_{n=0}^{\infty} V_{n}(r) P_{n}(\cos \theta) \\
V_{\text {out }}=\sum_{n=0}^{\infty} V_{n}(R)\left(\frac{R}{r}\right)^{n+1} P_{n}(\cos \theta)
\end{gathered}
$$


the set of partial differential equations (3.1-7) is transformed to an infinite set of ordinary differential equations. We note that (3.11) is the solution of (3.4) that is regular at infinity and satisfies the boundary condition (3.6).

Substituting the solution (3.8-11) into the equations (3.1-7) and using the appropriate relations between the Legendre functions and their recurrence relations [13] the variable $\theta$ can be eliminated.

Using the dimensionless quantities,

$$
\begin{array}{cll}
\tau=r / R, & h=H_{z} / 2 \pi M_{s}, & h_{r}=\omega / 2 \pi M_{s} \gamma_{0}, \quad u=V / 2 \pi M_{s} R_{0} \\
& R_{0}=\sqrt{A} / M_{s}, & A=C / 2, \quad S=R / R_{0}
\end{array}
$$

and the notations

$$
L_{n}=\frac{\mathrm{d}}{\mathrm{d} \tau}+\frac{n+1}{\tau}, \quad M_{n}=\frac{\mathrm{d}}{\mathrm{d} \tau}-\frac{n}{\tau}
$$

the infinite set of ordinary differential equations, in which the set of equations $(3.1-3)$ is reduced, is given by:

$$
\begin{array}{cc}
\left(L_{n+1} M_{n}-\pi S^{2} h\right) B_{n}(\tau)+\pi S^{2} h_{r} A_{n}(\tau)=0, \quad n \geq 1 & \\
\left(L_{n+1} M_{n}-\pi S^{2} h\right) A_{n}(\tau)+\pi S^{2} h_{r} B_{n}(\tau)=\pi S\left(M_{n-1} \frac{u_{n-1}(\tau)}{2 n-1}-L_{n+1} \frac{u_{n+1}(\tau)}{2 n+1}\right), & n \geq 1 \\
L_{n+1} M_{n} u_{n}(\tau)=2 S\left[\frac{(n+1)(n+2)}{(2 n+3)} L_{n+1} A_{n+1}(\tau)-\frac{n(n-1)}{(2 n-1)} M_{n-1} A_{n-1}(\tau)\right], & n \geq 0
\end{array}
$$

with the following boundary conditions on $\tau=1$,

$$
\begin{gathered}
\frac{\mathrm{d} A_{n}}{\mathrm{~d} \tau}=\frac{\mathrm{d} B_{n}}{\mathrm{~d} \tau}=0, \quad n \geq 1 \\
L_{n} u_{n}(\tau)=2 S\left[\frac{(n+1)(n+2)}{(2 n+3)} A_{n+1}(\tau)-\frac{n(n-1)}{(2 n-1)} A_{n-1}(\tau)\right], \quad n \geq 0 .
\end{gathered}
$$

We seek a solution of the set of equations (3.12-14) in the following form:

$$
\begin{aligned}
& A_{n}(\tau)=\sum_{k=1}^{\infty} a_{n, k} j_{n}\left(\mu_{k} \tau\right), \quad n \geq 1 \\
& B_{n}(\tau)=\sum_{k=1}^{\infty} b_{n, k} j_{n}\left(\mu_{k} \tau\right), \quad n \geq 1 \\
& u_{n}(\tau)=\sum_{k=1}^{\infty} c_{n, k} j_{n}\left(\mu_{k} \tau\right), \quad n \geqslant 0
\end{aligned}
$$

where $j_{n}(x)$ are the $n$-th spherical Bessel functions and $a_{n, k}, b_{n, k}, c_{n, k}$ and $\mu_{k}$ are unknown coefficients.

By substitution of the above trial solution into equations (3.12-14) we obtain

$$
\begin{array}{cc}
\sum_{k=1}^{\infty}\left[\pi S^{2} h_{r} a_{n, k}-\left(\mu_{k}^{2}+\pi S^{2} h\right) b_{n, k}\right] j_{n}\left(\mu_{k} \tau\right)=0, \quad n \geq 1 & \\
\sum_{k=1}^{\infty}\left[\pi S^{2} h_{r} b_{n, k}-\left(\mu_{k}^{2}+\pi S^{2} h\right) a_{n, k}+\pi S \mu_{k}\left(\frac{c_{n-1, k}}{2 n-1}+\frac{c_{n+1, k}}{2 n+3}\right)\right] j_{n}\left(\mu_{k} \tau\right)=0, & n \geq 1 \\
\sum_{k=1}^{\infty}\left[2 S \mu_{k}\left(\frac{(n+1)(n+2)}{(2 n+3)} a_{n+1, k}+\frac{n(n-1)}{(2 n-1)} a_{n-1, k}\right)+\mu_{k}^{2} c_{n, k}\right] j_{n}\left(\mu_{k} \tau\right)=0, & n \geq 0 .
\end{array}
$$


In order $(3.20-22)$ to be valid $\forall \tau$, the following relations have to be satisfied,

$$
\begin{array}{cl}
\pi S^{2} h_{r} a_{n, k}-\left(\mu_{k}^{2}+\pi S^{2} h\right) b_{n, k}=0, \quad n \geq 1, & k \geq 1 \\
\pi S^{2} h_{r} b_{n, k}-\left(\mu_{k}^{2}+\pi S^{2} h\right) a_{n, k}+\pi S \mu_{k}\left(\frac{c_{n-1, k}}{2 n-1}+\frac{c_{n+1, k}}{2 n+3}\right)=0, & n \geq 1, \quad k \geq 1 \\
2 S\left[\frac{(n+1)(n+2)}{(2 n+3)} a_{n+1, k}+\frac{n(n-1)}{(2 n-1)} a_{n-1, k}\right]+\mu_{k} c_{n, k}=0, & n \geq 0, \quad k \geq 1 .
\end{array}
$$

From (3.23) and (3.24) we obtain

and

$$
b_{n, k}=\frac{\pi S^{2} h_{r}}{\mu_{k}^{2}+\pi S^{2} h} a_{n, k}, \quad n \geq 1, \quad k \geq 1, \quad \mu_{k}^{2}+\pi S^{2} h \neq 0
$$

$$
c_{n, k}=-\frac{2 S}{\mu_{k}}\left[\frac{(n+1)(n+2)}{(2 n+3)} a_{n+1, k}+\frac{n(n-1)}{(2 n-1)} a_{n-1, k}\right], \quad n \geq 1, \quad k \geq 1,
$$

respectively, and (3.25) leads to the following recurrence relation

$$
a_{n+2, k}=\beta_{n k} a_{n, k}+\xi_{n} a_{n-2, k}, \quad n \geq 1, \quad k \geq 1
$$

where $a_{-m, k}=0$ for $m=0,1,2, \ldots$ and

$$
\begin{gathered}
\beta_{n k}=\frac{(2 n+3)(2 n+5)}{2 \pi S^{2}(n+2)(n+3)}\left[\frac{\left(\pi S^{2} h_{r}\right)^{2}}{\mu_{k}^{2}+\pi S^{2} h}-\left(\mu_{k}^{2}+\pi S^{2} h\right)-4 \pi S^{2} \frac{n(n+1)}{(2 n-1)(2 n+3)}\right] \\
\xi_{n}=-\frac{(n-1)(n-2)(2 n+3)(2 n+5)}{(2 n-1)(2 n-3)(n+2)(n+3)} .
\end{gathered}
$$

In view of the solution (3.17-19), the boundary conditions (3.15) and (3.16) become

$$
\begin{gathered}
\sum_{k=1}^{\infty}\left\{\begin{array}{l}
\left.a_{n, k}\right\} \\
b_{n, k}
\end{array}\right\} \mu_{k} j_{n}\left(\mu_{k}\right)=0, \quad n \geq 1 \\
\sum_{k=1}^{\infty}\left[2 S\left(\frac{(n+1)(n+2)}{2 n+3} j_{n+1}\left(\mu_{k}\right) a_{n+1, k}-\frac{n(n-1)}{2 n-1} j_{n-1}\left(\mu_{k}\right) a_{n-1, k}\right)\right. \\
\left.-\mu_{k} j_{n-1}\left(\mu_{k}\right) c_{n, k}\right]=0, \quad n \geq 0 .
\end{gathered}
$$

Taking into account (3.26), (3.27) and the recurrence relation,

$$
j_{n}^{\prime}(x)=j_{n-1}(x)-\frac{n+1}{x} j_{n}(x)
$$

where ()$^{\prime}=d / d x$, the conditions (3.29) and (3.30) are written as:

and

$$
\begin{array}{rlrl}
\sum_{k=1}^{\infty} a_{n, k}\left[\mu_{k} j_{n-1}\left(\mu_{k}\right)-(n+1) j_{n}\left(\mu_{k}\right)\right] & =0, & & n \geq 1 \\
h_{r} \sum_{k=1}^{\infty} a_{n, k} \frac{\mu_{k} j_{n-1}\left(\mu_{k}\right)-(n+1) j_{n}\left(\mu_{k}\right)}{\mu_{k}^{2}+\pi S^{2} h} & =0, & n \geq 1,
\end{array}
$$

$$
2 S \sum_{k=1}^{\infty} \frac{(n+1)(n+2)(2 n+1)}{2 n+3} \frac{j_{n}\left(\mu_{k}\right)}{\mu_{k}} a_{n+1, k}=0, \quad n \geq 0,
$$

respectively. Equation (3.33) can equivalently be written as

$$
\sum_{k=1}^{\infty} \frac{j_{n-1}\left(\mu_{k}\right)}{\mu_{k}} a_{n, k}=0, \quad n \geq 1 .
$$


The satisfaction of the boundary conditions (3.31), (3.32) and (3.34) is guaranteed when the following quadratic form:

$$
\sum_{n=1}^{\infty}\left[(\text { Eq. (3.31) })^{2}+(\text { Eq. }(3.32))^{2}+(\text { Eq. }(3.34))^{2}\right]
$$

vanishes. This condition can be written as

$$
f\left(\mu_{k}, a_{1, k}, a_{2, k}, h, h_{r}, S\right)=0
$$

since from the recurrence relation (3.28), every $a_{n, k}$ can be expressed in terms of $a_{1, k}$ and $a_{2, k}$. The equation (3.36) is solved numerically, by using an optimization technique proposed by Papageorgiou et al. $[8,9]$.

\section{NUMERICAL RESULTS AND DISCUSSION}

A numerical solution of equation (3.36) was carried out by the aid of the optimization package MERLIN-2.1 [8,9]. For given $S$ and $h_{r}$, equation (3.36) determines the eigenvalue $h$ for finite $n$ and $k$. The number of terms in the sums was increased until the difference between consecutive eigenvalues of $(h)$ was negligible. For a matter of convenience we chose $\mu_{k}$ to be the positive zeros of $j_{1}^{\prime}(x)=0$. The numerical calculation of $\mu_{k}$ was carried out by the use of function FindRoot, of the package Mathematica [14].

The results of the computation are shown in Figs 1-4. The variation of the resonance field $(h)$ with the resonance frequency $\left(h_{r}\right)$ is plotted in Fig. 1 and with the size parameter $(S)$ in Fig. 2 , for various values of $h$ and $h_{r}$, respectively. It is noted that $h$ is always larger than the nucleation field of the static problem and $h_{r}$ is real, because only then the precession about the reference state is stable. Values of $h$ smaller than the static nucleation field have no physical significance because the corresponding oscillations occur around an unstable reference state [12]. It is also seen that the zero frequency limit $\left(h_{r}=0\right)$ of the resonance field coincides with the nucleation field of the static problem, which, in the case under discussion, corresponds to the curling mode [15-17]. The resonance field is a decreasing function of $S$, a behaviour at least qualitatively the same with the experimental results [2], though far from the corresponding size range $(R \ll 1 \mathrm{~mm})[6]$.

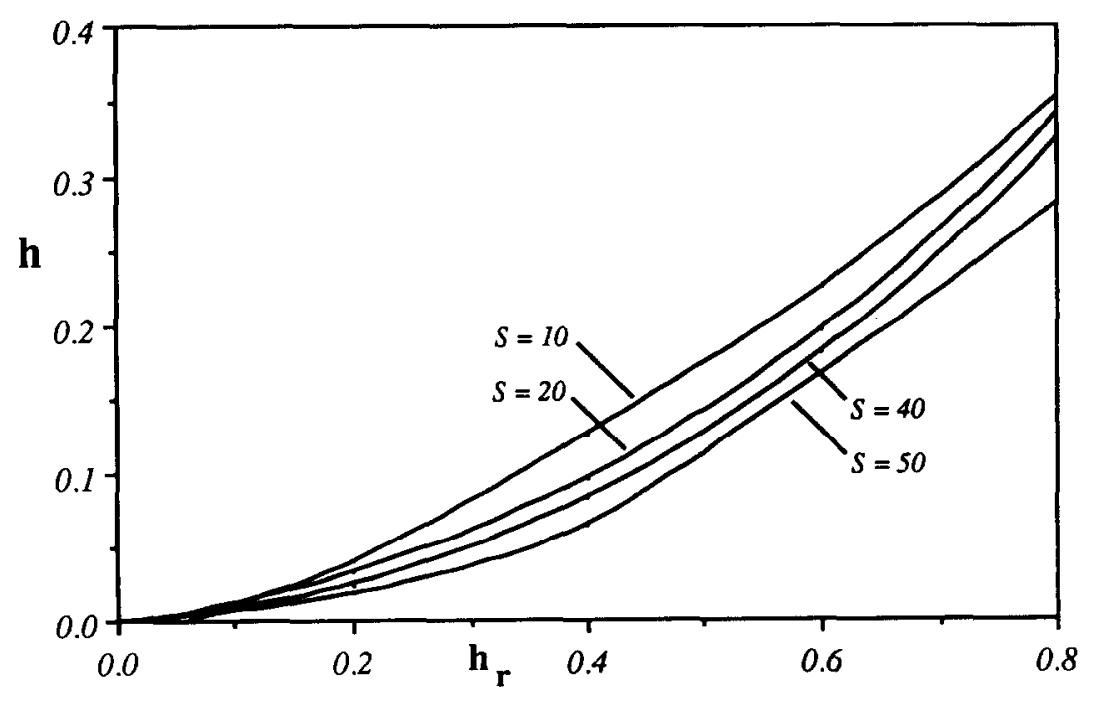

Fig. 1. Variation of $h$ with $h_{r}$. 


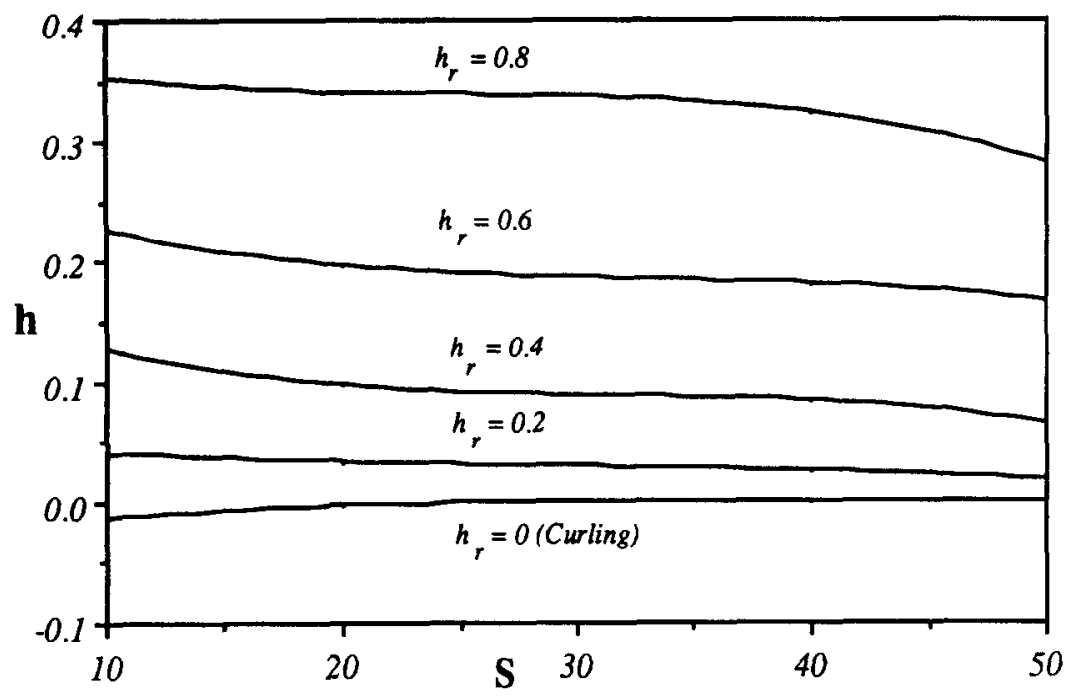

Fig. 2. Variation of $h$ with $S$.

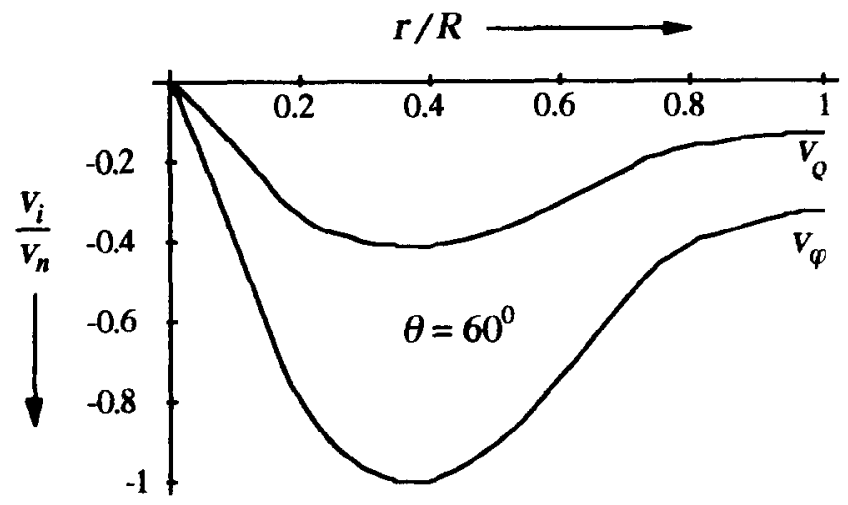

Fig. 3. Reduced components $v_{\varrho}, v_{\varphi}$ as a function of the reduced radial distance $r / R$ for $h=0.324, h_{r}=0.8$, $S \stackrel{\varphi}{=} 40$ for $i=\varrho, \varphi$ and $v_{n}=\max \left(\left|v_{\varrho}\right|, \mid v_{\varphi}\right)$.

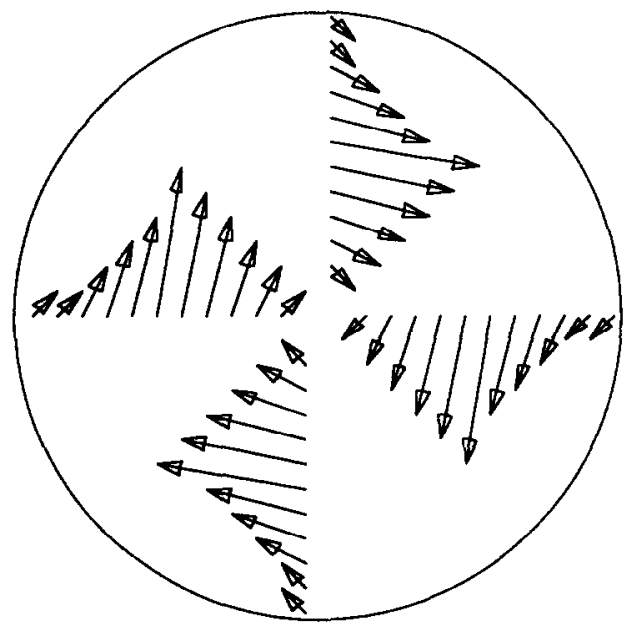

Fig. 4. Configuration of the transverse magnetization for $\theta=60^{\circ}, h=0.324, h_{r}=0.8, S=40$. 
For the case of magnetite $\left(\mathrm{Fe}_{3} \mathrm{O}_{4}\right)$ with $A \simeq 10^{-7} \mathrm{erg} / \mathrm{cm}, M_{s} \simeq 4.5 \times 10^{5} \mathrm{~A} / \mathrm{m}\left(R_{0}=1.98 \mathrm{~nm}\right)$, $\gamma_{0}=1.105 \times 10^{5} \mathrm{~g} \times \mathrm{m} /$ Asec and $g \simeq 2.001$ we obtain for $10 \leq S \leq 50 \Leftrightarrow 19.8 \mathrm{~nm} \leq R \leq 99 \mathrm{~nm}$ that the resonance field $H_{z}$ varies as $0.019 \leq h \leq 0.352 \Leftrightarrow 676 \mathrm{Oe} \leq H_{z} \leq 12.523 \mathrm{kOe}$ for frequencies $f\left(\omega=2 \pi f \Rightarrow h_{r}=f / M_{s} \gamma_{0}\right), 0.2 \leq h_{r} \leq 0.8 \Leftrightarrow 19.9 \mathrm{GHz} \leq f \leq 79.6 \mathrm{GHz}$, or wavelengths $\lambda$, in the following range: $3.8 \mathrm{~mm} \leq \lambda \leq 1.5 \mathrm{~cm}$.

The proposed analysis, in comparison to that presented by Aharoni [7], has the advantage of computing the eigenvectors of the problem and it is therefore possible to plot the shape of the magnetization configuration in the material. The results for $\theta=60^{\circ}, h_{r}=0.8, S=40$ and $h=0.324$ are plotted in Fig. 3. The vertical axis corresponds to the normalized components $v_{e}$ and $v_{\phi}$ and the horizontal to the distance from the center of the sphere. It is also seen that the dominant contribution is of the circumferential component of the transverse magnetization vector. A more realistic picture of the vector field of the transverse magnetization is shown in Fig. 4. It is obvious that it looks like some kind of curling. That is why it could be named generalized curling.

\section{REFERENCES}

[1] L. R. BICKFORD, Phys. Rev. 78, 449 (1950).

[2] R. RAJAGOPALAN and J. K. FURDYNA, Phys. Rev. B 39(4), 2532 (1989).

[3] L. R. WALKER, Phys. Rev. 105(2), 390 (1957).

[4] V. PAPAEFTHYMIOU et al., J. Appl. Phys. 67(9), 4487 (1990),

[5] S. NAFIS et al., IEEE Trans. Magn. MAG-25(5), 3641 (1989).

[6] A. AHARONI, J. Appl. Phys. 69(11), 7762 (1991).

[7] A. AHARONI, J. Appl. Phys. 64(11), 6434 (1988).

[8] D. G. PAPAGEORGIOU, C. S. CHASSAPIS and I. E. LAGARIS, Comput. Phys. Commun. 52, 241 (1989); D. G. PAPAGEORGIOU and I. E. LAGARIS, Comput. Phys. Commun. 58, 119 (1990).

[9] G. A. EVAGElAKIS, J. P. RIZOS, I. E. LAGARIS and D. DEMETROPOULOS, Comput. Phys. Commun. 46, 401 (1987).

[10] II. F. TIERSTEN, J. Math. Phys. 5, 1 (1964).

[11] C. V. MASSALAS, Int. J. Engng Sci. 29, 10 (1991).

[12] W. F. BROWN, Micromagnetics. Wiley (1963).

[13] C. V. MASSALAS, Special Functions. University of Ioannina (in Greek) (1988).

[14] S. WOLFRAM, Mathematica (A System of Doing Mathematics by Computer). Addison-Wesley (1991).

[15] A. AHARONI, J. Appl. Phys. 30(4), 70S (1959).

[16] P. A. VOlTAiRAS and C. V. MASSALAS, Mech. Res. Commun. 19(2), 135 (1992).

[17] P. A. VOLTAIRAS and C. V. MASSALAS, Proc. Fourth ISEM Symp. on Nonlinear Phenomena in Electromagnetic Fields, Nagoya, 26-29 January 1992. Elsevier Studies in Applied Electromagnetics in Materials 3 (Edited by T. FURUHASHI and Y. UCHIKAWA), pp. 477-480 (1992). 